OPEN ACCESS

Edited by:

Shu Yuan,

Sichuan Agricultural University, China

Reviewed by:

Matthew Terry,

University of Southampton, UK Toshinori Kinoshita,

Nagoya University, Japan

*Correspondence:

Nobuyoshi Mochizuki

mochizuki@physiol.bot.kyoto-u.ac.jp

Specialty section: This article was submitted to Plant Physiology, a section of the journal

Frontiers in Plant Science

Received: 29 July 2016 Accepted: 20 October 2016 Published: 07 November 2016

Citation:

Ibata H, Nagatani $A$ and Mochizuki N (2016) CHLH/GUN5 Function in

Tetrapyrrole Metabolism is Correlated with Plastid Signaling but not $A B A$

Responses in Guard Cells.

Front. Plant Sci. 7:1650. doi: 10.3389/fpls.2016.01650

\section{CHLH/GUN5 Function in Tetrapyrrole Metabolism Is Correlated with Plastid Signaling but not ABA Responses in Guard Cells}

\author{
Harue Ibata, Akira Nagatani and Nobuyoshi Mochizuki * \\ Department of Botany, Graduate School of Science, Kyoto University, Kyoto, Japan
}

Expression of Photosynthesis-Associated Nuclear Genes (PhANGs) is controlled by environmental stimuli and plastid-derived signals ("plastid signals") transmitting the developmental and functional status of plastids to the nucleus. Arabidopsis genomes uncoupled (gun) mutants exhibit defects in plastid signaling, leading to ectopic expression of PhANGs in the absence of chloroplast development. GUN5 encodes the plastid-localized Mg-chelatase enzyme subunit (CHLH), and recent studies suggest that $\mathrm{CHLH}$ is a multifunctional protein involved in tetrapyrrole biosynthesis, plastid signaling and $\mathrm{ABA}$ responses in guard cells. To understand the basis of $\mathrm{CHLH}$ multifunctionality, we investigated 15 gun5 missense mutant alleles and transgenic lines expressing a series of truncated CHLH proteins in a severe gun5 allele (cch) background (tCHLHs, 10 different versions). Here, we show that Mg-chelatase function and plastid signaling are generally correlated; in contrast, based on the analysis of the gun 5 missense mutant alleles, ABA-regulated stomatal control is distinct from these two other functions. We found that none of the $\mathrm{tCHLHs}$ could restore plastid-signaling or Mg-chelatase functions. Additionally, we found that both the $\mathrm{C}$-terminal half and $\mathrm{N}$-terminal half of $\mathrm{CHLH}$ function in ABA-induced stomatal movement.

Keywords: GUN5, CHLH, Mg-chelatase, tetrapyrrole, plastid signal, stomatal ABA response

\section{INTRODUCTION}

CHLH (also called GENOMES UNCOUPLED 5, GUN5) is a multifunctional protein involved in chlorophyll biosynthesis, plastid-to-nucleus signaling, and ABA responses. CHLH is a plastidlocalized protein that has been studied as the largest $(\mathrm{H})$ subunit of Mg-chelatase, which catalyzes the conversion of Protoporphyrin IX (Proto) to Mg-Protoporphyrin IX (MgProto), a key step in the tetrapyrrole biosynthesis pathway in plants (Tanaka et al., 2011). Mg-chelatase is composed of three catalytic subunits, CHLH/GUN5, CHLD, and CHLI, and a regulatory subunit, GUN4. CHLH is the porphyrin binding subunit, and CHLD-CHLI confers Mg-ATPase activity (Gibson et al., 1995; Willows et al., 1996). CHLH protein sequences are well conserved from photosynthetic bacteria to land plants. In addition, the protein structure of $\mathrm{CHLH}$ has been studied using singleparticle reconstruction and small-angle X-ray scattering (Qian et al., 2012), and the crystal structure of Synechocystis PCC6803 CHLH (SynCHLH) was recently solved at $2.5 \AA$ resolution (Chen et al., 2015). SynCHLH is thought to consist of six domains (I-VI, Supplementary Figures 1, 14), with small amino (N)-terminal domains (domains I and II) that form a "head" and "neck" structure, 
followed by a cage-like assembly (domains III-VI). It was reported that CHLH predominantly exists as a monomer in solution (Qian et al., 2012), whereas a loosely bound CHLH dimer was observed in the crystal. The dimerization interface are domains I and V, which is consistent with a previous study that removal of the $\mathrm{N}$-terminal 159 residues of $T$. elongates $\mathrm{ChlH}$ facilitates a monomeric state (Adams et al., 2014). The porphyrin-binding internal pocket is proposed to be located at the interface between domains III and V, a region with the most conserved residues. The Arabidopsis mutants cch and gun5-1 have respectively a Pro to Leu substitution at residue 642 (P642L) and an Ala to Val substitution at residue 990 (A990V). These positions map to P595 and A942 in the SynCHLH protein (Supplementary Figure 1), which are located respectively in domain III and at the junction between domains III and V (Chen et al., 2015). Because the cch and gun5-1 mutant CHLH proteins can bind Proto but are catalytically inactive, it has been proposed that these mutations may introduce spatial hindrance and interfere with chelation (Davison and Hunter, 2011).

Plastid-to-nucleus retrograde signaling (also called "plastid signaling") controls diverse aspects of cellular activity such as plastid development, response to abiotic stress, hormone signaling, and shoot and fruit development (Chan et al., 2016). Several different signals and pathways have been identified, including tetrapyrroles, isoprenes, phosphoadenosines, carotenoid derivatives, reactive oxygen species, and proteins. The GENOMES UNCOUPLED (GUN) pathway is triggered by the arrest of plastid development under photooxidative stress induced by the carotenoid biosynthesis inhibitor norflurazon (NF) or under the inhibition of plastid translation caused by lincomycin (Susek et al., 1993; Gray et al., 1995). Under such conditions, transcription of photosynthesis-associated nuclear genes (PhANGs) such as light-harvesting chlorophyll a/b binding protein 1 (Lhcb1) is severely repressed. Genetic studies using Arabidopsis have led to the discovery of gun mutants that exhibit derepression of $L h c b 1^{*} 2$ under these conditions (hereafter called the "gun phenotype") (Susek et al., 1993; Mochizuki et al., 2001). Of six GUN genes, GUN2-GUN6 are involved in tetrapyrrole metabolism, suggesting that tetrapyrrole is key for such signaling. GUN2 (HY1), GUN3 (HY2), and GUN6 (FC1) encode a heme oxygenase, phytochromobilin synthase, and Fe-chelatase, respectively (Mochizuki et al., 2001; Woodson et al., 2011). As mentioned above, GUN4 and GUN5 encode the regulatory and porphyrin-binding subunits of $\mathrm{Mg}$-chelatase, respectively (Mochizuki et al., 2001; Larkin et al., 2003). GUN1 is a plastid-localized pentatricopeptide repeat-small MutS-related protein (PPR-SMR) that is proposed to serve as a central hub of GUN plastid signaling (Koussevitzky et al., 2007). A recent report suggests that the GUN1 protein interacts with plastid ribosomal proteins and early tetrapyrrole biosynthesis enzymes (Tadini et al., 2016). Although several lines of evidence suggest that Mg-protoporphyrin IX and heme status are key in plastid signaling, the exact signaling mechanism remains elusive (Mochizuki et al., 2001, 2008; Strand et al., 2003; Moulin et al., 2008).

CHLH is also reported to be involved in responses to abscisic acid (ABA) in Arabidopsis. Recent studies reveal that the family containing pyrabactin resistance (PYR), pyrabactin resistance 1-like (PYL), and regulatory component of ABA receptor $(\mathrm{RCAR})$ proteins are potent candidates for $\mathrm{ABA}$ receptors that regulate SnRK2 family protein kinases through type 2C protein phosphatases (PP2Cs) (Cutler et al., 2010). In a different experimental approach, CHLH was identified as an ABA-binding protein, and subsequent biochemical and genetic analyses suggested that CHLH binds to ABA and mediates the $\mathrm{ABA}$ signal in the regulation of stomatal aperture, seed germination, root elongation, and induction of ABA-responsive genes (Zhang et al., 2002; Shen et al., 2006). However, whether $\mathrm{CHLH}$ binds to ABA and has functions other than stomatal ABA responsiveness remain unclear (Shen et al., 2006; Muller and Hansson, 2009; Wu et al., 2009; Tsuzuki et al., 2011). In a subsequent study, CHLI1, but not CHLD and GUN4, was shown to be involved in ABA signaling resulting in stomatal movement in Arabidopsis and tobacco (Tsuzuki et al., 2011; Du et al., 2012). Moreover, it has been reported that knockdown of CHLM (encoding the enzyme responsible for the step after $\mathrm{Mg}$-chelatase) also leads to ABA insensitivity in stomatal movement (Tomiyama et al., 2014). Furthermore, as a reduction in $\mathrm{Mg}$-chelatase activity was observed in CHLM antisense RNAoverexpressing tobacco (Alawady and Grimm, 2005), defects in the CHLM protein may affect the function of the Mg-chelatase complex. These findings suggest that Mg-chelatase and CHLH are involved in the stomatal response to ABA.

Structural and functional studies have been carried out in Arabidopsis to elucidate the molecular nature of CHLH in ABA signaling (Wu et al., 2009; Shang et al., 2010). It has been proposed that the CHLH protein spans the outer and inner envelopes, with the $\mathrm{N}$ - and C-termini exposed to the cytoplasm (Wu et al., 2009). A group of WRKY transcription repressors are suggested to interact with the cytoplasmic C-terminal region of CHLH (Shang et al., 2010). When CHLH binds to ABA, WRKY proteins are recruited to $\mathrm{CHLH}$ and ABA-responsive genes are induced in the nucleus. For these experiments, transgenic Arabidopsis lines expressing truncated $\mathrm{CHLH}$ in wild type and the $c c h$ mutant background were produced and tested for sensitivity to ABA. With regard to ABA sensitivity in stomatal movement, both CHLH fragments with an internal deletion (line C370, a.a. $1-120+631-999$; full-length CHLH is 1381 a.a.) and C- and N-terminally truncated CHLH (a.a. 1-772 and 6311381, respectively) conferred ABA responsiveness. The authors concluded that the C-terminal half of CHLH plays a central role in $\mathrm{ABA}$ signaling and that the $\mathrm{N}$-terminal region is required for proper regulation of the $\mathrm{C}$-terminal half. It should be noted that line C370 complemented tetrapyrrole and chlorophyll synthesis deficiencies in the $c c h$ mutant.

New candidates for the signaling components downstream of CHLH-mediated ABA signaling have been suggested in recent reports. By yeast two-hybrid system using a.a. 348-1038 or 6921381 of CHLH protein as a bait, CPN20 (plastid co-chaperonin) and SnRK2.6/OST1 (sucrose non-fermenting 1 (SNF1)-related protein kinase 2 family/open stomata 1) have been found as interaction partners, respectively (Zhang et al., 2013; Liang et al., 2015). SOAR1, which encodes a pentatricopeptide repeat (PPR) protein localizing in both the cytosol and nucleus, has 
been identified by a suppressor screening of ABA-hypersensitive phenotype in overexpressor of the truncated (a.a. 631-999) CHLH (Mei et al., 2014). Although it has been suggested that these genes are involved in ABA signaling including the regulation of stomatal aperture, seed germination, and seedling growth, the functions in $\mathrm{Mg}$-chelatase and plastid signaling have not been examined.

Despite knowledge of the detailed structure as well as functional analyses, the relationship between the function of the $\mathrm{CHLH}$ protein as a Mg-chelatase enzyme and component of plastid and ABA signaling is unclear to date. Because one of the difficulties in studying CHLH is that the null mutation results in an albino and seedling-lethal phenotype, we performed a new screen for gun mutants, isolated a number of missense mutant alleles of gun 5 and performed a detailed phenotypic analysis. To compare functionality in different physiological responses, we also produced Arabidopsis transgenic lines expressing a series of truncated CHLH/GUN5s based on previous works (Wu et al., 2009).

\section{MATERIALS AND METHODS}

\section{Plant Material and Growth Conditions}

The Arabidopsis thaliana strains used were all derived from the Col-0 ecotype. gun5 mutant alleles were isolated from the transgenic line CL3-5, as described below. gun5-1 and cch (conditional chlorina, Mochizuki et al., 2001) were backcrossed to CL3-5. In all the experiments, CL3-5 was used as wild type. rtl1 (rapid transpiration in detached leaves 1, Tsuzuki et al., 2011) was provided by Dr. T. Kinoshita (Nagoya Univ.), and the R1290C line (in the gll background) was provided by Dr. A. Takemiya (Yamaguchi Univ.) Plants were grown in soil with 1000-fold-diluted Hyponex or on Murashige and Skoog Plant Salt Mixture (Wako) agar medium containing 2\% (w/v) sucrose at $23^{\circ} \mathrm{C}$. For photobleaching, seedlings were grown for 5 days on MS agar medium supplemented with $2 \%$ sucrose and $2.5 \mu \mathrm{M}$ Norflurazon (NF, Sandoz Pharmaceutical) under continuous white light $\left(100 \mu \mathrm{mol} / \mathrm{m}^{2} / \mathrm{s}\right)$. For measurement of stomatal aperture, plants were grown on vermiculite under long-day condition [16 h fluorescent light $\left(50 \mu \mathrm{mol} / \mathrm{m}^{2} / \mathrm{s}\right) / 8 \mathrm{~h}$ dark cycle] at $23^{\circ} \mathrm{C}$ and approximately $85 \% \mathrm{RH}$. For testing inhibitory effect of $\mathrm{ABA}$ on seed germination and seedling growth, seeds were sown on MS agar medium containing $2 \%(\mathrm{w} / \mathrm{v})$ sucrose in the absence or presence of $0.5 \mu \mathrm{M}$ $\mathrm{ABA}$, and the plates were incubated as described above after stratification.

\section{Production of the Lhcb1*2 Promoter-Luciferase Line CL3-5}

The $5^{\prime}$ upstream region $(1.1 \mathrm{~kb})$ of the $L h c b 1^{*} 2$ gene was amplified from Co-0 genomic DNA by PCR with CAB3pro-Fw0 and CAB3pro-Rv0' primers. The fragment was digested with HindIII/NcoI and ligated into pGL3basic (Promega), resulting in the pGL3basic/Lhcb1*2pro:LUC plasmid. The HindIII-EcoRI (blunted) region of the pBI-Hyg/35S-NosT vector (Yamaguchi et al., 1999) was inserted into the HindIII-KpnI (blunted) site in pCGN1547 (McBride and Summerfelt, 1990), which resulted in pCGN1547/35S-nosT. The CMV 35S promoter was removed from pGCN1547/35S-nosT by HindIII-XbaI digestion and substituted with the HindIII-XbaI fragment of Lhcb1*2pro-LUC from pGL3basic/Lhcb1*2pro-LUC to generate pGCN/CAB3-LUC. The resulting plasmid was used for the plant transformation as described below. The primer sequences are listed in Supplementary Table 1.

\section{EMS Treatment and Mutant Screening}

CL3-5 seeds were treated with $0.2 \%(\mathrm{v} / \mathrm{v}) \mathrm{EMS}$ at $25^{\circ} \mathrm{C}$ for $16 \mathrm{~h}$. To obtain M2 seeds, the seeds were washed thoroughly, sown on vermiculite, and grown under continuous fluorescent light at $23^{\circ} \mathrm{C}$. Approximately 66,000 M2 seeds from 20,000 M1 plants were subjected to the following screen. Individual NF-treated seedlings were submerged in $0.1 \mathrm{mM}$ Luciferin (Calbiochem) and $0.01 \%$ Tween-80 in 96-well opaque titer plates. Bioluminescence was measured using a Microbeta scintillation counter (Perkin Elmer). Seedlings showing greater than 3-fold increase in luminescence compared to the average counts from the entire population was selected. By measuring luciferase luminescence in individual seedlings, we could detect mutants with a weaker phenotype than the previously reported gun mutants (Susek et al., 1993). The selected seedlings were transferred to MS plates to regenerate and set seeds. DNA was prepared from tissue from a portion of the M2 plants, and the genomic GUN5 region was amplified by PCR and sequenced using the primers listed in Supplementary Table 1.

\section{Production of tCHLH Constructs}

Truncated CHLH (tCHLH) lines were produced as follows. The $5^{\prime}$-upstream region $(1 \mathrm{~kb})$ of $C H L H$ was PCR amplified from Co-0 genomic DNA using primers CHLHpro-SL-1 and CHLHp3R. pGWB511 (Nakagawa et al., 2007) was amplified using primers pGWB514-SL-11 and GW500spacerF. These fragments were connected at the overlapping sequences by a SLiCE reaction (Zhang et al., 2012), resulting in pGWB511CHLHpro. pGWB511-CHLHpro was digested with KpnI and used for a SLiCE reaction with the tCHLH fragments prepared as described below. The tCHLH clones listed in Figure 5 were constructed as follows. Fragments A, B, C, D, H, I, J, and full-length were PCR amplified from the CHLH cDNA clone using the primers listed in Supplementary Table 1. Fragment E was produced by overlap extension PCR (Horton et al., 1989). We first amplified the $5^{\prime}$ fragment using CHLH-GWMet and CHLH-120_631-Rv and the $3^{\prime}$ fragment by separately using CHLH-120_631-Fw and CHLH-1381-Rv. These two PCR products were then mixed and PCR amplified for 5 cycles. After the addition of primers CHLH-GW-Met and CHLH-1381-Rv, PCR was further performed for 15 cycles to obtain fragment E. Fragment E was cloned into pENTR/D-TOPO (Invitrogen), resulting in pENTR-E. Fragments E, F, and G were amplified using pENTR-E as a template. All fragments were ligated to KpnI-digested pGWB511-CHLHpro by a SLiCE reaction, which resulting in p511-full and p511-A through p511-J. The plasmid was used for the plant transformation as described below. The primer sequences are listed in Supplementary Table 1. 


\section{Transformation of Agrobacterium and Plants}

The plasmid was introduced into Agrobacterium tumefaciens (GV3101::pMP90) by the freeze-thaw method (Chen et al., 1994), which was subsequently transferred to plants using floral dip method (Clough and Bent, 1998). Transgenic plants were selected on MS agar medium containing the relevant antibiotics. T3 or T4 homozygous plants were used in all experiments.

\section{RNA Extraction and RT-qPCR}

Total RNA was extracted from whole seedlings using the Sepazol RNA I super kit (Nacalai Tesque) following the manufacturer's instructions. cDNA was synthesized with oligo(dT) ${ }_{12-18}$ using Transcriptor first-strand synthesis kit (Roche) according to the manufacturer's instructions. Real-time PCR was performed using LightCycler 480 SYBR Green I Master (Roche) and a LightCycler 96 (Roche). The following standard thermal profile was used for all reactions: $95^{\circ} \mathrm{C}$ for $5 \mathrm{~min}$, followed by 40 cycles of $95^{\circ} \mathrm{C}$ for $5 \mathrm{~s}$, $55^{\circ} \mathrm{C}$ for $10 \mathrm{~s}$, and $72^{\circ} \mathrm{C}$ for $20 \mathrm{~s}$. The primer sequences are listed in Supplementary Table 1.

\section{Quantification of Protoporphyrin IX, Mg-Protoporphyrin IX, and Mg-Protoporphyrin IX Monomethylester}

Porphyrins were extracted according to Mochizuki et al. (2008), and Mg-Protoporphyrin IX (MgProto) and Mg-Protoporphyrin IX monomethylester (MgProtoMe) were detected at an excitation wavelength of $417 \mathrm{~nm}$ and emission at 595, nm with a $15-\mathrm{nm}$ band path. After the MgProtoMe peak was eluted, the detector was adjusted for the detection of Protoporphyrin IX (Proto, excitation, $402 \mathrm{~nm}$; emission $633 \mathrm{~nm}$ ). Standard curves were constructed with authentic standards purchased from Frontier Science.

\section{Quantification of Chlorophyll Levels}

Chlorophyll was extracted for $16 \mathrm{~h}$ in complete darkness at $4^{\circ} \mathrm{C}$ from 10 5-day-old seedlings using $\mathrm{N}, \mathrm{N}$-dimetylformamide. The extract was subjected to spectrophotometric measurements at 664, 647, and $750 \mathrm{~nm}$. The specific chlorophyll content was calculated using the equations of Porra et al. (1989) and normalized to the total fresh weight for each sample.

\section{Anti-Full-Length CHLH Antiserum Production}

A 6xHis-full-length CHLH protein was produced exactly as previously described (Wu et al., 2009). Briefly, cDNA encoding full-length $\mathrm{CHLH}$ was amplified by PCR using primers CHLHZ1-F and CHLH-Z1-R. The PCR product was digested with EcoRI and SalI and then cloned into pET48b(+) at the EcoRI and SalI sites. The recombinant CHLH protein was expressed in E. coli Rosetta gami2 (DE3) (Novagen) and purified on a $\mathrm{Ni}^{2+}$-NTA column, as described in the manual provided by the manufacturer (Qiagen). Antiserum against 6xHis-fulllength $\mathrm{CHLH}$ was raised by immunizing rabbits according to a standard protocol (Kiwa Laboratory Animals Co., Ltd). The primer sequences are listed in the Supplementary Table 1.

\section{Immunoblot Analysis}

Protein extraction from Arabidopsis seedlings was performed as described (Shen et al., 2006). A 10-20- $\mu$ g sample of protein was separated by $7.5 \%$ SDS-PAGE and blotted onto a nitrocellulose membrane. The rabbit anti-CHLH polyclonal antibody (see above) and a mouse anti-FLAG monoclonal antibody (Wako) were used as primary antibodies at a dilution of 1:5000. An anti-rabbit IgG antibody or anti-mouse IgG antibody conjugated with horseradish peroxidase (Promega) was used as a secondary antibody at a dilution of 1:5000. Quantitation of band strength was performed using Multi Gauge Ver3.0 (FUJIFILM, Japan).

\section{Preparation of Epidermal Peels for Measurement of Stomatal Aperture}

Intact epidermal peels were prepared according to the Perforated-tape Epidermal Detachment method (the PED method, Ibata et al., 2013). For experiments, fully expanded rosette leaves were harvested from 4- to 6-week-old Arabidopsis plants. For PED, Time Tape (Hirasawa) was used to hold the adaxial surface of the leaf, and Scotch Magic Tape (3M) perforated with a hole was used to detach the lower abaxial epidermis from the Arabidopsis leaves. The epidermal peel affixed to the tape was immediately submerged in an appropriate buffer or solution for subsequent experiments.

\section{Measurement of Stomatal Aperture}

Stomatal aperture was measured according to Inoue et al. (2008), with some modifications. Leaves were soaked in $3 \mathrm{ml} \mathrm{1/2} \mathrm{MS}$ medium and incubated in the dark for $16 \mathrm{~h}$ at $23^{\circ} \mathrm{C}$ to cause stomatal closure. The epidermal layers were prepared by the PED method, and the layers were maintained in $3 \mathrm{ml}$ of basal reaction buffer [5 mM MES-BTP (MES, 2-(N-morpholino) ethanesulfonic acid and BTP, 1,3- bis (tris (hydroxymethyl) methylamino) propane], $\mathrm{pH}$ 6.5, $50 \mu \mathrm{M} \mathrm{KCl}$, and $0.1 \mathrm{mM} \mathrm{CaCl}_{2}$ ). The samples were irradiated for $2.5 \mathrm{~h}$ at $23^{\circ} \mathrm{C}$ with red light at $50 \mu \mathrm{mol} / \mathrm{m}^{2} / \mathrm{s}$ (Red) or blue light at $10 \mu \mathrm{mol} / \mathrm{m}^{2} / \mathrm{s}$ supplemented with 50 $\mu \mathrm{mol} / \mathrm{m}^{2} / \mathrm{s}$ red light (Blue) in the absence or presence of 20 $\mu \mathrm{M}$ ABA. For ABA-induced stomatal closure, pre-illuminated epidermal tissues were incubated under blue light supplemented with red light, as described above, for $2.5 \mathrm{~h}$ with or without $20 \mu \mathrm{M}$ ABA. Images of the epidermis were captured using a microscope (BX51, Olympus) equipped with a digital camera (C11440, HAMAMATSU). The images were analyzed with ImageJ (NIH) software to obtain stomatal aperture data. Only visibly intact guard cells were subjected to stomatal aperture measurement. For each strain, the size of the stomatal aperture is expressed as the mean of 25 stomata with the standard deviation (SD).

\section{RESULTS}

\section{Screening for New gun5 Missense Mutant Alleles}

We screened for new gun 5 mutant alleles using a transgenic line harboring Lhcb1*2pro:LUC (CL3-5). We isolated 120 candidate lines showing elevated expression of the reporter in the presence 
of NF. Among these candidates, we identified 11 gun 5 mutant alleles by determining the genomic sequence of GUN5/CHLH regions (Figures 1A,B). These gun5 alleles were backcrossed to the parental CL3-5 line at least three times and used for further analysis. A mutant (R1290C) plant that exhibits abnormal leaf surface temperature due to the opened stomatal phenotype, kindly provided by Dr. Takemiya from a mutant collection (Takemiya et al., 2013), was also examined. Three previously reported gun5 mutant alleles (cch, rtll, and gun5-1) were also included in our analysis.

In the following sections, we evaluate the mutants for three different phenotypes: (1) gun phenotype-Lhcb1*2 expression; (2) Mg-chelatase phenotype-tetrapyrrole intermediate and chlorophyll levels in 5-day-old seedlings; and (3) ABA phenotype - light-dependent change in stomatal aperture in leaves of adult plants treated with ABA.

\section{Analysis of Plastid Signaling and Tetrapyrrole Intermediates and Chlorophyll Levels in New gun5 Mutants}

For evaluation of the gun phenotype, endogenous Lhcbl*2 mRNA levels were measured by RT-qPCR. Most of the mutant seedlings exhibited elevated $L h c b 1^{*} 2$ expression in the presence of NF, though a mutation near the N-terminal region (D235N) did not result in significantly elevated $L h c b 1^{*} 2$ expression compared to wild type (Figure 2A). However, the periphery of the N-terminus of CHLH appears to be crucial for CHLH function because S92F showed a strong gun phenotype. We then tested tetrapyrrole intermediate levels under the same condition $(+\mathrm{NF})$ and found significant correlation between $L h c b 1^{*} 2$ expression and tetrapyrrole levels (Figure 2, Supplementary Figures 3D-F). While the levels of Proto and MgProtoMe are positively and negatively correlated $(p<$ 0.001 ) to the level of $L h c b 1^{*} 2$ expression, respectively, we found a less significant correlation between the MgProto levels and the gun phenotype in the presence of NF, despite MgProto levels tending to be lower in the mutants compared to the wild type (Figure 2B, Supplementary Figure 3E), and gun phenotype $(+\mathrm{NF})$ showed significant negative correlation $(p<0.002)$ to the MgProto levels in the absence of NF (Figure 2A, Supplementary Figures 2B, 3B). Elevation of Proto and decrease of MgProto \& MgProtoMe in these mutants are the results of a defect in Mg-chelatase activity, which appears to be correlated to the gun phenotype as proposed previously (Strand et al., 2003). However, it is evident that mutations at the C-terminus (A1253V and R1290C) resulted in a normal level of tetrapyrrole intermediate with a weak but distinct gun phenotype. Chl a levels (-NF) were tied in to the $L h c b 1^{*} 2$ mRNAs in the absence of NF $(p<0.05)$ (Supplementary Figures 2A,C, 3G), although no significant link between $\mathrm{Chl}$ levels and gun phenotypes $(+\mathrm{NF})$ in the mutants were found (Supplementary Figure $3 \mathbf{H}$ ).

\section{Stomatal ABA Response in gun5 Mutants}

Light-induced stomatal opening and the inhibitory effect of externally applied ABA were tested in the mutants. Plants for only four mutant alleles, including the previously reported cch and $r t l 1$, were insensitive to ABA in close-to-open and open-to-close assays (Figures 3A,B). Most other lines (9 alleles) responded normally to $\mathrm{ABA}$ with regard to light-induced stomatal opening (Supplementary Figure 4). In addition to ABA-insensitive alleles, we found two mutations (P450L and A1253V) displaying a constitutively closed stomatal phenotype (Figures 4A,B, indicated as "homo"). Overexpression of CHLH in guard cells driven by an epidermis-specific promoter (CER6pro) is reported to exhibit a similar phenotype (Tsuzuki et al., 2013). Unlike the CER6pro-CHLH-GFP overexpressor, $\mathrm{P} 450 \mathrm{~L}$ and $\mathrm{A} 1253 \mathrm{~V}$ did not show a hypersensitive phenotype in response to $20 \mu \mathrm{M} \mathrm{ABA}$ (Figures $4 \mathbf{A}, \mathbf{B}$ ), and only a trace level of CHLH protein was found for the P450L mutant (Supplementary Figure 5A). Analysis of stomatal phenotype in the backcrossed F1 plants indicates that the P450L and A1253V mutations are recessive (Figures $\mathbf{4 A , B}$, indicated as "hetero"). As the constitutively closed stomatal phenotype may be caused by a lack of plasma membrane-localized $\mathrm{H}^{+}$-ATPase activation, we treated these mutants with fusicoccin, an activator of $\mathrm{H}^{+}$ATPase that induces stomatal opening without light stimulation. The stomatal apertures of the P450L and A1253V mutants were restored to wild type when treated with fusicoccin (Figure 4C), suggesting these mutants have defects in the upstream regulatory pathway leading to the activation of the stomatal plasma membrane localized- $\mathrm{H}^{+}$-ATPase. In view of the disparities in previous reports on other ABA-related phenotypes of gun5 mutants (ABA resistance on seed germination and growth) (Shen et al., 2006; Muller and Hansson, 2009; Wu et al., 2009; Tsuzuki et al., 2011), we tested the cch and rtl1 mutants for the seed germination and seedling growth in the presence of $0.5 \mu \mathrm{M}$ $\mathrm{ABA}$. We found equal sensitivity to ABA between the mutants and the wild type (Supplementary Figure 6). This data suggests that CHLH function in ABA-related function is limited to the stomatal guard cells.

\section{CHLH Protein Level in gun5 Mutants}

Differences in phenotype among plants with the mutant alleles might be due to variation in CHLH protein accumulation and protein function. Hence, we examined CHLH protein levels in the mutants by Western blotting using an anti$\mathrm{CHLH}$ antibody. CHLH is greatly reduced in wild type in the presence of NF (Supplementary Figures 5B,C) In contrast, the majority of the mutants had equivalent or even higher levels of CHLH protein than wild type in the absence or presence of NF. These results suggest that a complete absence of the CHLH protein is not the cause of the observed phenotypes (Supplementary Figures 5B,C). Further support to this observation comes from previous studies where links between the strength of the gun phenotype and increments in mRNA level in gun5 mutant as well as regulation of $\mathrm{CHLH} / \mathrm{GUN5}$ gene by plastid signaling have been drawn (Moulin et al., 2008 and Supplementary Figure 7). It should be noted that clear differences in CHLH protein accumulation were found among the mutants with a similar gun phenotype, e.g., V679M and L690L (rtl1) (Figure 2A), but the CHLH protein levels significantly differed 


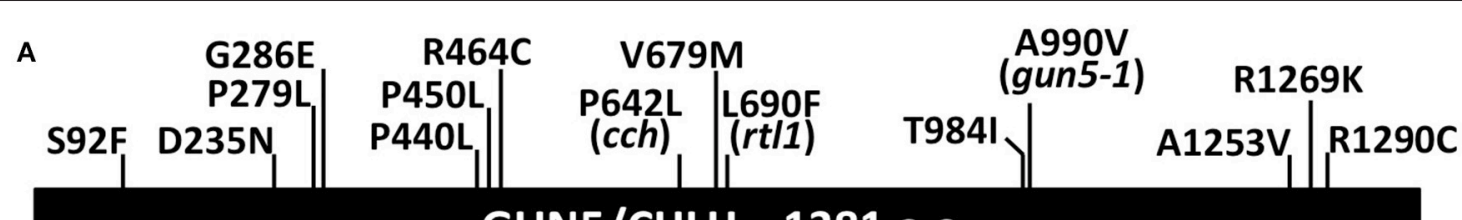

GUN5/CHLH 1381 a.a.

B

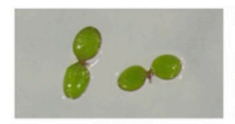

S92F

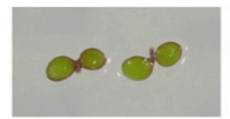

P450L

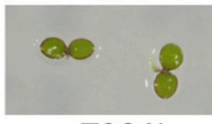

T984I

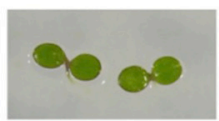

D235N

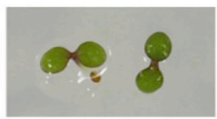

R464C

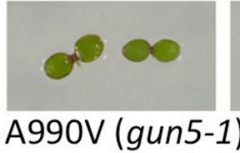

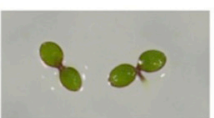

P279L

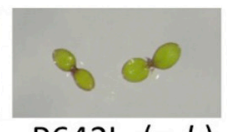

P642L (cch)

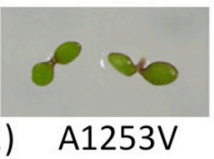

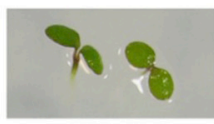

G268E

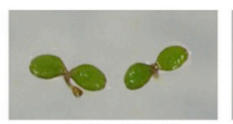

V679M

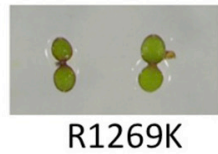

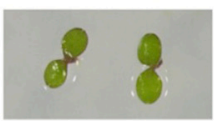

P440L

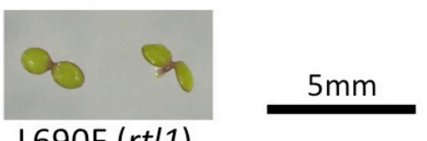

L690F (rt/1)

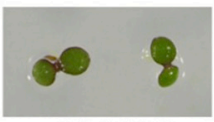

R1290C

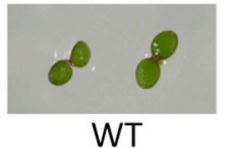

FIGURE 1 | gun5 mutant alleles used in this study. (A) Schematic diagram of the GUN5/CHLH protein and the positions of amino acid substitutions of the mutant alleles used in this study. Wild type amino acids and positions and mutant amino acids are indicated. (B) gun5 mutant seedlings were grown on MS medium supplemented with $2 \%$ sucrose under $100 \mu \mathrm{mol} / \mathrm{m}^{2} / \mathrm{s}$ white light for 5 days. The scale bar is $5 \mathrm{~mm}$.

among the lines (Supplementary Figure 5C). Likewise, no significant relationship was observed for the $\mathrm{CHLH}$ protein level and the stomatal ABA phenotype (Figures 3, 4A,B, Supplementary Figures 4, 5A).

\section{Expression of Truncated CHLH Proteins (tCHLHs)}

We employed a different approach to gain insight into the domain structure of CHLH responsible for the individual physiological functions. Transgenic lines expressing truncated $\mathrm{CHLH}$ proteins have been used to delineate domains related to ABA responsiveness ( $\mathrm{Wu}$ et al., 2009). Accordingly, we designed 11 different constructs with either full-length CHLH, a C-terminal, N-terminal, or internal deletion of CHLH, the sGFP-tag at the C-terminus and driven by the cauliflower mosaic virus $35 \mathrm{~S}$ promoter (35Spro). These constructs were transformed into the $c c h$ mutant; however, most hygromycinresistant T1 seedlings were albino and seedling lethal, which was most likely due to co-suppression or gene silencing. We thus replaced 35Spro with the endogenous promoter of $\mathrm{CHLH}$ and introduced FLAG as a C-terminal tag instead of sGFP (Figure 5). Utilizing these new constructs, we were able to obtain a reasonable number of transgenic plants, in which the expression of truncated CHLH (tCHLH) was tested by Western blotting using anti-CHLH and anti-FLAG antibodies. As shown in Supplementary Figures 8A-B, 9A-D, all lines expressed fulllength GUN5-FLAG (CHLH-full) or tCHLH-FLAG at least during the seedling stage. Based on the band intensities using the anti-FLAG antibody (tCHLH-B as a reference), most tCHLH lines expressed an approximately 2-fold amount of the protein compared to wild type. Accumulation of tCHLH-H and tCHLHJ was especially low, and these lines were detected on overloaded blots in a separate experiment (Supplementary Figures 9C,D). Some lines exhibited reduced protein accumulation at the adult stage (Supplementary Figures 10A,B). We selected two or three representative lines among each $\mathrm{tCHLH}$ lines for the analysis below.

\section{Plastid Signaling, Mg-Chelatase and ABA Phenotype in tCHLH Lines}

tCHLH lines were first examined for the gun phenotype in the presence of NF. The control line, CHLH-full, exhibited a wild type or even reduced $L h c b 1^{*} 2$ expression, complementing the gun phenotype (Figure 6A). In contrast, all other lines failed to complement and some showed enhanced gun phenotype (Figure 6A, Supplementary Figure 11A). CHLH-full did not significantly alter the gene expression levels of $L h c b 1^{*} 2$, while some of the tCHLH lines showed elevated expression of $L h c b 1^{*} 2$ in the absence of NF (Supplementary Figures 12C,D). Elevation of $L h c b 1^{*} 2$ expression in the absence or presence of NF observed in some tCHLH lines suggests dominant negative effect of these tCHLHs to $c c h$ mutation. Regarding the levels of tetrapyrroles (Proto, MgProto, and MgProtoMe), CHLH-full was able to complement $c c h$ in the absence or presence of NF (Figure 6B, Supplementary Figure 12A). On the other hand, the other tCHLH lines did not restore tetrapyrrole levels in the absence or presence of NF (Figure 6B, Supplementary Figures 11B, 12A,B), though statistically significant differences were detected 

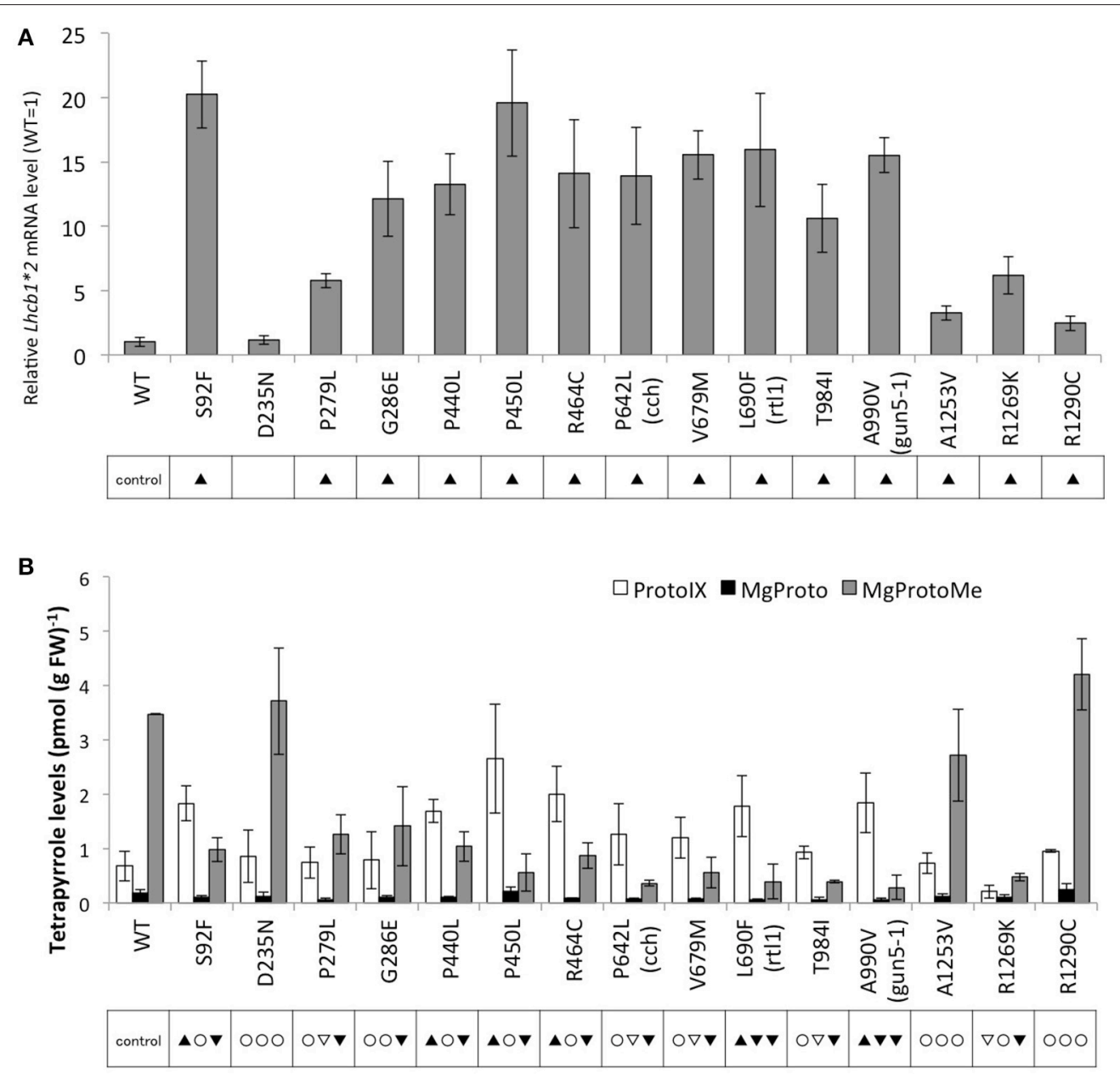

FIGURE 2 | Comparison of gun and tetrapyrrole phenotypes in wild type and gun5 mutant alleles in the presence of NF. Plants were grown on MS medium supplemented with $2 \%$ sucrose and $2.5 \mu \mathrm{M} \mathrm{NF}$ for 5 days under continuous white light $\left(100 \mu \mathrm{mol} / \mathrm{m}^{2} / \mathrm{s}\right)$. (A) Lhcb1 mRNA levels in wild type and gun 5 mutants grown in the presence of NF. Lhcb1 mRNA levels were quantified and normalized to TUB2 mRNA levels by RT-qPCR, as described in Materials and Methods. Data shown are the mean $\pm \mathrm{SD}(n=3)$, and the WT level is presented as 1.0. (B) Proto, MgProto, and MgProtoMe levels in wild type and gun5 mutants in the presence of NF. Tetrapyrrole was extracted and quantified by HPLC and normalized to fresh weight, as described in Materials and Methods. Proto (white bar), MgProto (black), and MgProtoMe (gray) levels are presented. Data shown are the mean $\pm \mathrm{SD}(n \geqq 3)$. In (A) and (B), statistical significance was determined by Student's $t$-test. The black triangle and the inverted black triangle indicate significant differences $(p<0.05)$ of the average value with the higher value or lower value compared to that of the wild type control, respectively. For (B), significance in Proto, MgProto, MgProtoMe are indicated from left to right in each box, respectively. The symbols are the same as (A), except that the white triangle and inverted white triangle indicate weaker significant differences $(p<0.057)$ of the average value with the higher value or lower value compared to that of the wild type control, respectively, and the white circle indicates no significant difference.

in some lines when compared to the parental $c c h$ line. Likewise, chlorophyll-less phenotypes showed similar trends. CHLH-full restored chlorophyll content to wild type levels, while tCHLH lines showed equivalent or even lower abundance compared to the parental cch mutant (Supplementary Figures 11C,D). We were unable to reproduce the previous report that the expression of regions corresponding to tCHLH-A and tCHLH-G could complement both chlorophyll and tetrapyrrole phenotypes (ABARn and C370, respectively in $\mathrm{Wu}$ et al., 2009).

We then tested the tCHLH lines for sensitivity to ABA with respect to stomatal movement. Contrary to the results presented above, all tCHLH lines, except for tCHLH-H and
tCHLH-J, restored the ABA sensitivity of guard cells, as shown in Supplementary Figure 13. The complementation failure observed in tCHLH-H and tCHLH-J may due to the lower abundance of tCHLH compared to other lines. In a previous study (Wu et al., 2009), the fragment containing a.a. 1-772 was unable to complement the $c$ ch stomatal phenotype, a result that led to the proposal that the C-terminal half, especially the region containing a.a. 631-999, is responsible for $\mathrm{ABA}$ sensitivity. However, we did observe recovery of ABA sensitivity in the tCHLH-B, $-\mathrm{C}$, and -D lines, though the tCHLH-D does not overlap with the 631-999 region. These data suggest that the regions that complement the guard cell ABA-insensitive phenotype in the $c c h$ mutant is not limited to the C-terminal 

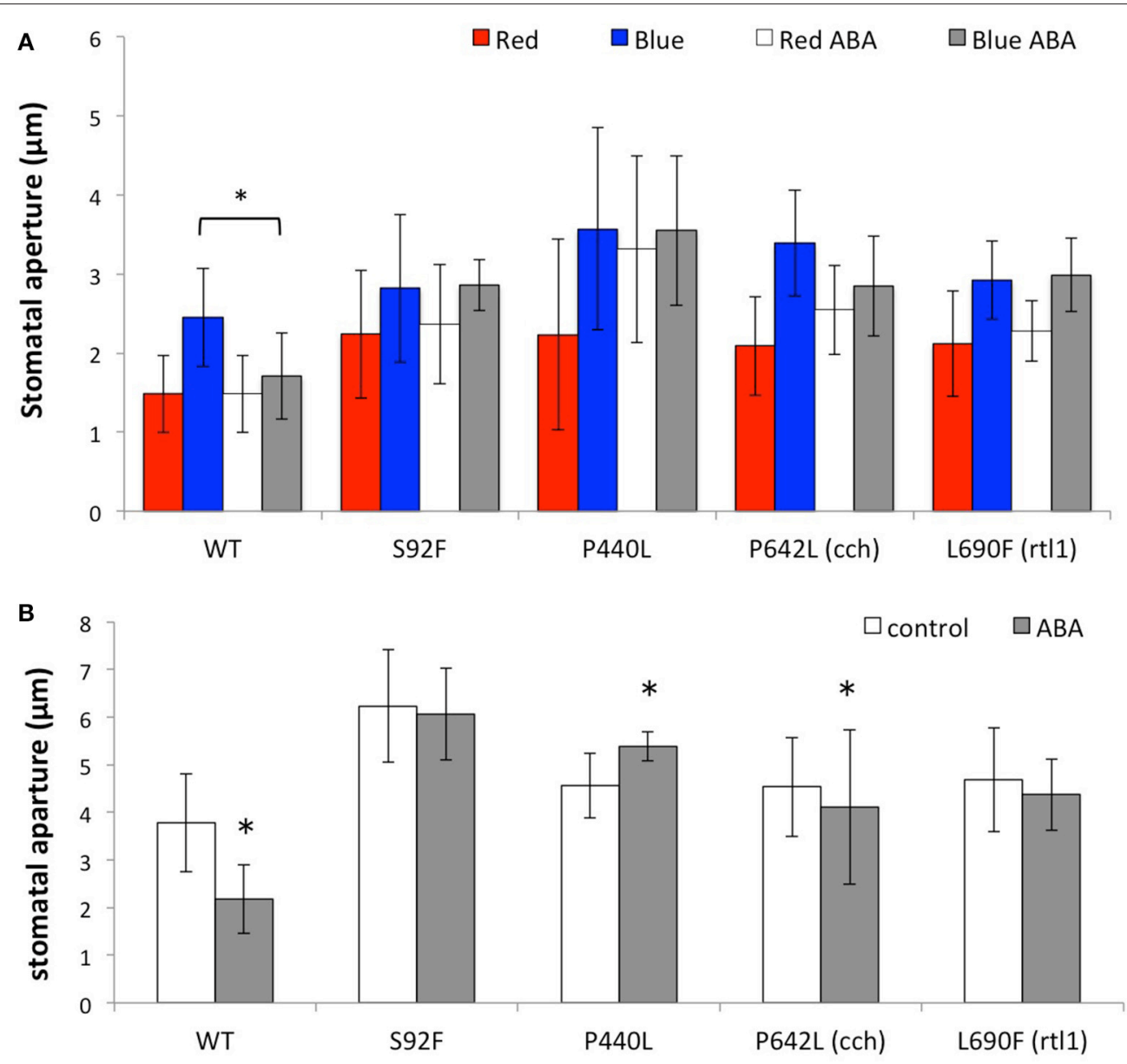

FIGURE 3 | Characterization of ABA-insensitive gun5 mutants. (A) Effect of ABA on light-induced stomatal opening in WT, S92F, P440L, P642L (cch), and L690F (rt/1) mutant plants. Epidermal tissues from dark-adapted plants were incubated under the following conditions for $2.5 \mathrm{~h}: \mathrm{red} \mathrm{light} \mathrm{at} 50 \mu \mathrm{mol} / \mathrm{m}^{2} / \mathrm{s}$ in the absence (red bar) or presence (white bar) of $20 \mu \mathrm{M} \mathrm{ABA}$ (+ABA); blue light at $10 \mu \mathrm{mol} / \mathrm{m}^{2} / \mathrm{s}$ with background red light at $50 \mu \mathrm{mol} / \mathrm{m}^{2} / \mathrm{s}$ in the absence (blue) or presence (gray) of $20 \mu \mathrm{M}$ ABA. (B) ABA-induced stomatal closure in wild type and mutants. Pre-illuminated epidermal tissues were incubated under blue light supplemented with red light for $2.5 \mathrm{~h}$ with (gray bar) without (white) $20 \mu \mathrm{M}$ ABA. Data are presented as the mean $\pm \mathrm{SD}(n=25)$. Statistical significance was determined by Student's $t$-test. ${ }^{*} p<0.05$.

half of the CHLH protein but that the N-terminus also confers an equivalent ability (see the summary in Figure 5).

\section{DISCUSSION}

Different degrees of phenotype strength among gun5 alleles have been reported, which suggests that specific regions (domains) of the CHLH protein correspond to functions of the $\mathrm{Mg}$ chelatase subunit, plastid-to-nucleus signaling, and guard cell ABA responses (Mochizuki et al., 2001; Shen et al., 2006; Wu et al., 2009; Shang et al., 2010). To further understand the molecular nature of CHLH protein, we collected 12 newly identified and three previously characterized gun5 missense mutant alleles and compared them with regard to phenotype. We also analyzed transgenic lines expressing a series of truncated $\mathrm{CHLH}$ proteins in $c c h$ mutant background. The summary of the analysis is shown in Figure 5 and Supplementary Figure 14.

\section{Analysis of Plastid Signaling, Mg-Chelatase, and ABA Response in gun5 Mutant Alleles}

In this study, we established that Proto and MgProtoMe levels in the presence of NF are positively and negatively correlated to the severity of gun phenotype in gun 5 mutants. Albeit the abundance of MgProto, a direct product of Mgchelatase, is less correlated to the phenotype. The accumulation of Proto and reduction of $\operatorname{MgProto}(\mathrm{Me})$ can be explained by the attenuation of $\mathrm{Mg}$-chelatase activity in gun5 mutants. Moderate reduction of MgProto might involve a coordinated attenuation of both $\mathrm{Mg}$-protoporphyrin IX methyltransferase and $\mathrm{Mg}$-chelatase activities, although such co-regulation has not been reported. Taken together, these data support the hypotheses that: (a) $\operatorname{MgProto}(\mathrm{Me})$ functions as a negative plastid signal (Strand et al., 2003), and (b) that defect in the metabolic flow to the Mg-branch may increase the flow into the Fe-branch, 


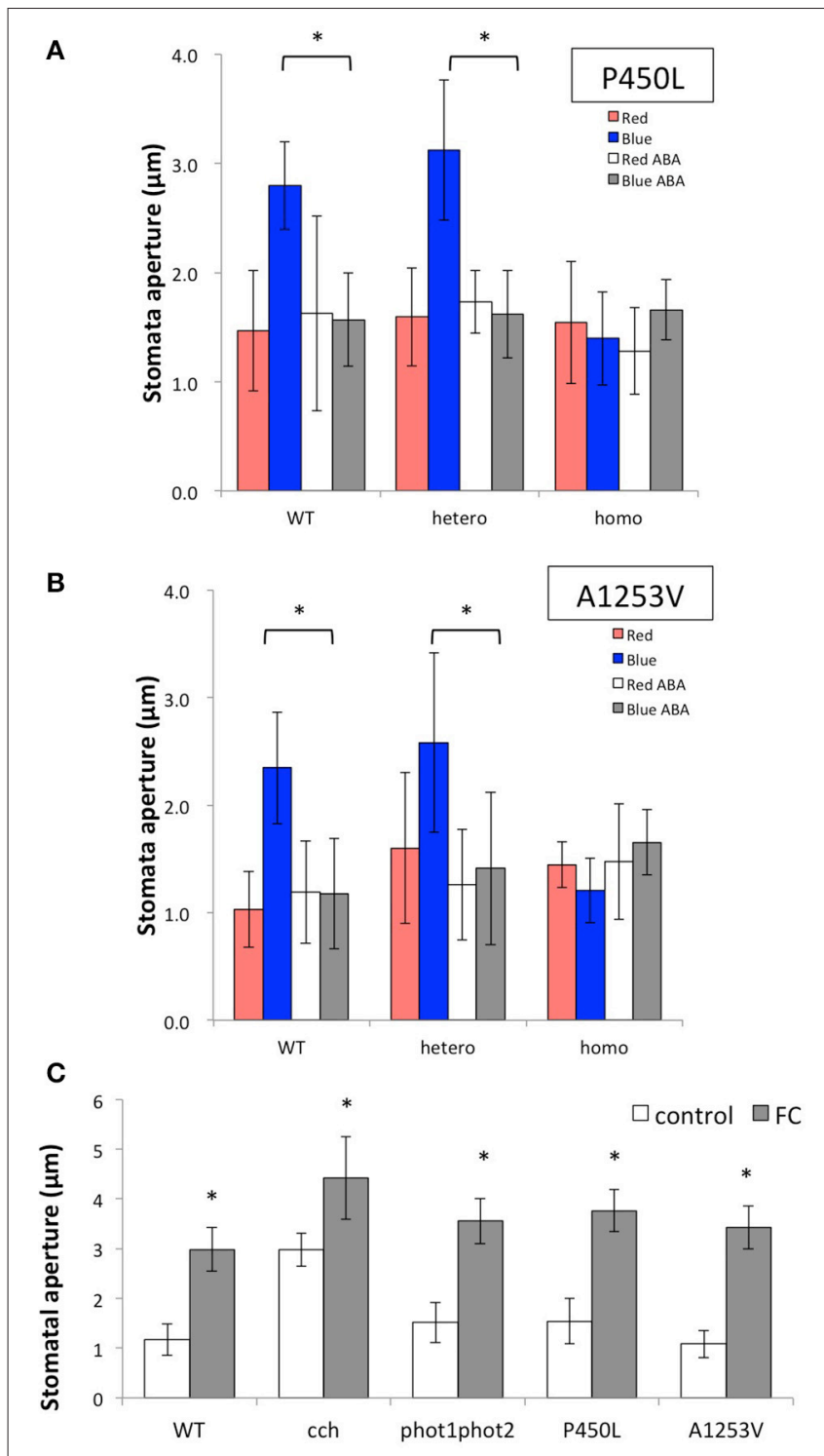

FIGURE 4 | Characterization of stomatal phenotype in P450L and A1253V mutants. Effect of ABA on light-induced stomatal opening in backcrossed F2 progeny of (A) P450L and (B) A1253V mutants. Wild type (WT), heterozygous (hetero), and homozygous (homo) segregants were subjected to the analysis described in Figure $\mathbf{3 A}$. Epidermal tissues from dark-adapted plants were incubated under the following conditions for $2.5 \mathrm{~h}$ red light at $50 \mu \mathrm{mol} / \mathrm{m}^{2} / \mathrm{s}$ in the absence (red bar) or presence (white bar) of $20 \mu \mathrm{M} \mathrm{ABA}(+\mathrm{ABA})$; blue light at $10 \mu \mathrm{mol} / \mathrm{m}^{2} / \mathrm{s}$ with background red light at $50 \mu \mathrm{mol} / \mathrm{m}^{2} / \mathrm{s}$ in the absence (blue) or presence (gray) of $20 \mu \mathrm{M}$ ABA. Data are presented as the mean $\pm \mathrm{SD}(n=25)$. (C) Effect of fusicoccin on light-induced stomatal opening in P450L and A1253V mutants. Epidermal tissues from dark-adapted plants were incubated under the following conditions for $2.5 \mathrm{~h}$ : blue light at $10 \mu \mathrm{mol} / \mathrm{m}^{2} / \mathrm{s}$ with background red light at $50 \mu \mathrm{mol} / \mathrm{m}^{2} / \mathrm{s}$ in the absence (white) or presence (gray) of $10 \mu \mathrm{M}$ fusicoccin. Data are presented as the mean \pm SD $(n=25)$. Statistical significance was determined by Student's $t$-test. ${ }^{*} p<0.05$.

and incremented heme level considered a positive plastid signal (Woodson et al., 2011; Terry and Smith, 2013). However, there are some exceptions. Mutations A1253V and R1290C at the Cterminus resulted in a normal level of tetrapyrrole intermediates with a weak but distinct gun phenotype. Although steady state levels of tetrapyrroles are not significantly changed in these mutants, they may have altered heme levels or a transient change in MgProto(Me) content as previously reported (Zhang et al., 2011). Future time-resolving profiling of tetrapyrroles including MgProto(Me) and heme may clarify our understanding the plastid signaling mechanism derived from tetrapyrrole metabolism and its intermediates.

Mutation in the central region of the CHLH protein, which corresponds to the C-terminal half of domains II and III in SynCHLH (Chen et al., 2015), has a strong impact on both Mgchelatase activity and plastid signaling; nonetheless, as discussed below, regions important for CHLH function are also present around the N- and C-termini, such as residue S92, R1269, and R1290 (Figure 2, Supplementary Figure 14). Based on structural aspects of the CHLH protein, it has been proposed that domains III and V comprise a buried pocket porphyrin-binding structure (Chen et al., 2015). Hence, mutations in the central region may alter the affinity of $\mathrm{CHLH}$ for porphyrins, which results in the attenuation of $\mathrm{Mg}$-chelatase activity and plastid signaling. However, one intriguing mutation, S92F, showed a strong gun phenotype comparable to that of mutations in the central region, though it has slight defects in tetrapyrrole levels. Because the predicted transit peptide region (a.a. 1-86) is likely to be removed upon entry into the plastid, S92 becomes situated near the N-terminus of CHLH (domain I in SynCHLH). According to the crystallographic analysis of SynCHLH, domains $\mathrm{I}$ and $\mathrm{V}$ form a dimerization interface; accordingly, S92F might interfere with the interaction between CHLH molecules (Supplementary Figure 14). Although an enhancing effect of the N-terminal fragment was previously reported using a reconstructed Synechocystis Mg-chelatase reaction (Sirijovski et al., 2008), the present study reports the first indication of the importance of the CHLH N-terminus to Mg-chelatase activity and plastid signaling in vivo. The residues at C-terminal region, A1253, R1269, and R1290, appear to be important for plastid signaling though they have less contribution to Mg-chelatase activity. Because the domains containing these residues (domain I \& the end of V to VI) locate outer region of the $\mathrm{CHLH}$ protein, the mutation in these residues may interfere with the interaction of other components such as CHLI, CHLD, and GUN4 that are involved in plastid signaling (Strand et al., 2003; Huang and Li, 2009; Adhikari et al., 2011).

Contrary to the chlorophyll and plastid signaling phenotypes, mutations affecting the stomatal ABA response had no clear relationship with these two phenotypes. For instance, G286E, R464C, and T984I are newly identified mutations with no ABA phenotype, even though they result in a relatively strong gun phenotype (Figure 2A, Supplementary Figures 4, 14A). Conversely, A1253V exhibits defects in ABA response but causes a weak gun phenotype. Among the six alleles that showed stomatal phenotypes, P462L (cch) and L690F ( $r t l 1)$ are located in the region corresponding to the ABA-binding core ( $\mathrm{Wu}$ et al., 2009), whereas the remaining four mutations are located in different regions of the $\mathrm{CHLH}$ protein, regions that have not previously been suggested to be involved in ABA signaling. We identified two novel mutations (P450L and A1253V) that 


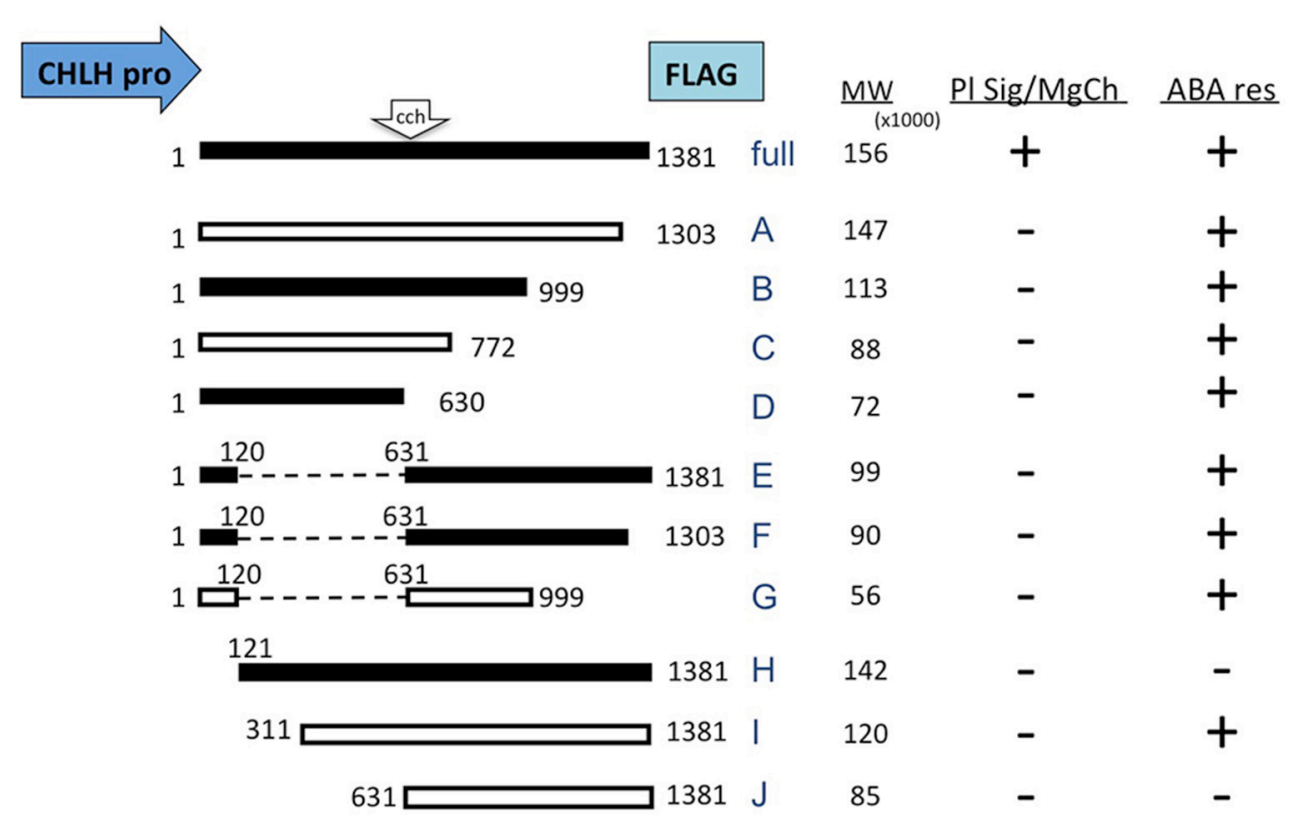

FIGURE 5 | Diagram of CHLH-full and truncated CHLH (tCHLH) constructs and a summary of phenotype analysis. The constructs presented as white boxes contain the same fragments used in a previous work (Wu et al., 2009), and the constructs presented as black boxes were designed in this study. All the constructs carry the endogenous CHLH promoter $(1.1 \mathrm{~kb})$ and a C-terminal FLAG tag. The position of the cch mutation (P642L) is indicated at the top of the diagrams. The line IDs (full and A-J) and calculated molecular weights (parts per thousand) are indicated at the right of each construct. Internal deletions in tCHLH-E, $-F$, and $-G$ are presented as dashed lines. A summary of phenotype assessments of plastid signaling and Mg-chelatase (PI sig/MgCh) activity and ABA response of stomatal movement (ABA res) is presented at the right of the diagrams. "+"; complemented, "-"; not complemented.

showed constitutively closed stomatal phenotype and appeared to have defect in the signal transduction pathway leading to the activation of $\mathrm{H}^{+}$-ATPase (Figure 4). A similar stomatal phenotype was observed when the CHLH-GFP fusion protein was overexpressed in guard cells under the epidermis-specific CER6 promoter (Tsuzuki et al., 2013). However, this does not appear to be the case for P450L and A1253V because we did not detect a significant increase in $\mathrm{CHLH}$ protein levels in these mutants (Supplementary Figure 5A) and genetic analysis of these mutants clearly showed that the mutations are both recessive (Figures 4A,B). For one intriguing mutation, V679M, the substitution of Val with Met results in a perfect match with the SynCHLH sequence (see Supplementary Figure 1). However, this "SynCHLH"-type substitution causes AtCHLH deficiency in plastid signaling and Mg-chelatase activity (Figures 2A,B, Supplementary Figures 2A,B). As V679 (M632 in SynCHLH) is located close to the residues that form the internal pocket, the internal pocket structure might be greatly affected by this substitution, even though the substitution is "conserved."

We were unable to identify gun5 mutants for domain IV, and only one mutation $(\mathrm{A} 1253 \mathrm{~V})$ at the peripheral region of domain $\mathrm{V}$ was found. Mutation in this region may cause severe defects in Mg-chelatase activity and therefore be seedling lethal. The analysis of such difficult regions may be facilitated using inducible deactivation of CHLH activity as described previously (Schlicke et al., 2014).

\section{Analysis of Plastid Signaling, Mg-Chelatase, and ABA Response in tCHLH Lines}

As an additional experiment, we adopted a gain-of-function approach by expressing truncated CHLH (tCHLH) in the cch mutant background and assessed the three physiological functions. Transgenic lines expressing full-length CHLH (CHLH-full) completely complemented the cch mutation in all three phenotypes (Figure 6, Supplementary Figures 11C, 12A). All other tCHLH lines were unable to complement the phenotypes. Some lines even showed dominant negative effects on the chlorophyll and gun phenotype, suggesting tCHLH interference of CHLH protein function in $c$ ch mutant. Contrary to our results, it was previously reported that a.a. 1-1303 and 1-120+631-999 (corresponding to tCHLH-A and tCHLH-G, respectively) can complement the chlorophyll phenotype of $c c h$ (Wu et al., 2009). This difference in results may be due to the relatively lower expression level of tCHLHs compared to that in the previous report. Contrary to the chlorophyll and plastid signaling phenotypes, the C-terminal (tCHLH-A to -D) and the middle+C-terminal deletion lines (tCHLH-E, -F, and -G), and an N-terminal deleted line (tCHLH-I) recovered guard cell sensitivity to ABA (Supplementary Figure 13). It should be noted that both tCHLH-C and tCHLH-D could rescue the ABA phenotype of $c c h$, even though they do not contain the region (a.a. 631-999) proposed to play a central role in ABA signaling (Wu et al., 2009). This finding suggests that either the 

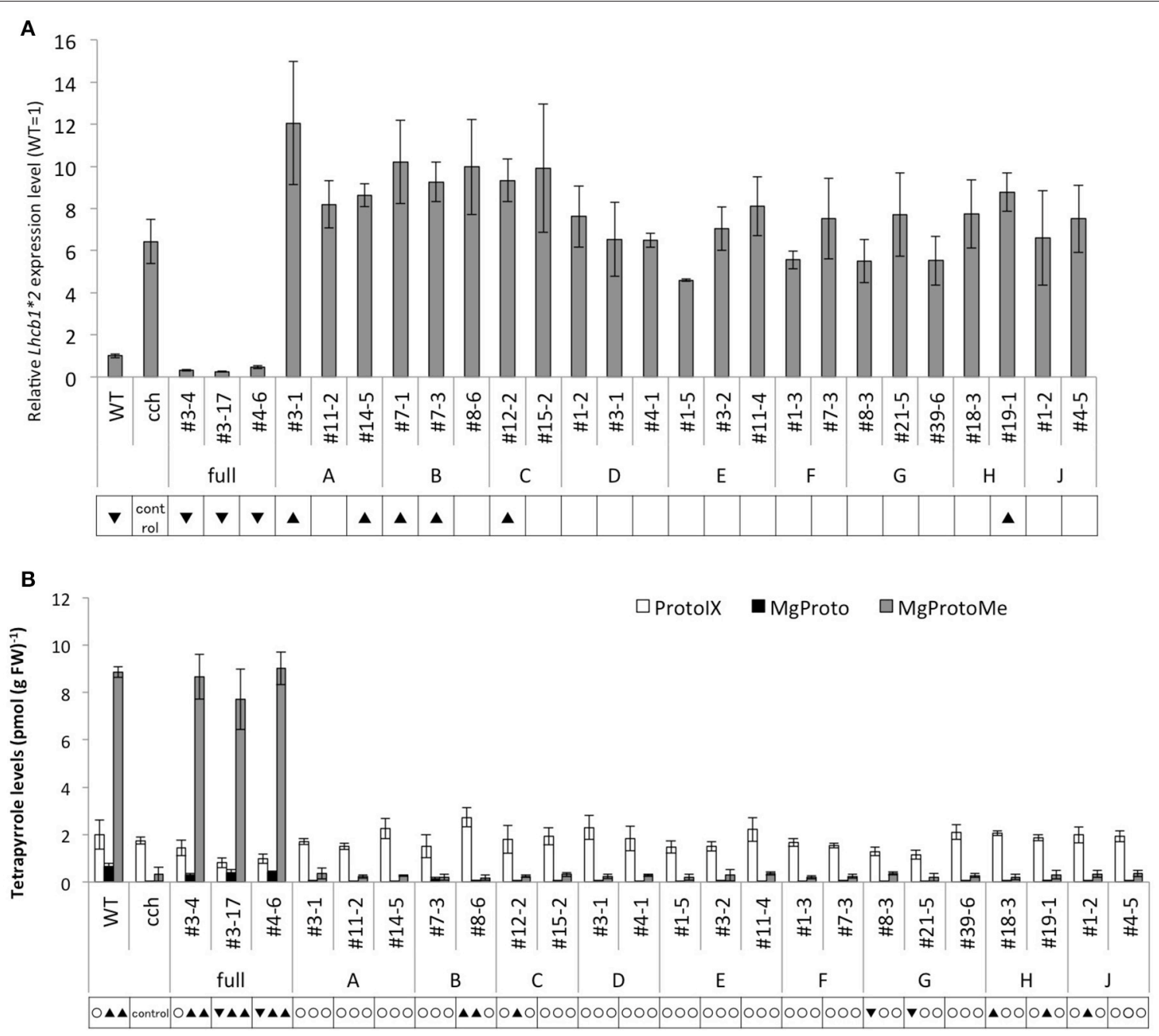

FIGURE 6 | Comparison of gun and tetrapyrrole phenotypes in wild type, cch, and tCHLH-transgenic plants. Plants were grown on MS medium supplemented with $2 \%$ sucrose and $2.5 \mu \mathrm{M} \mathrm{NF}$ for 5 days under continuous white light $\left(100 \mu \mathrm{mol} / \mathrm{m}^{2} / \mathrm{s}\right)$. (A) $L$ hcb1 mRNA levels in wild type and tCHLH plants grown in the presence of NF. Lhcb1 mRNA levels were quantified and normalized to TUB2 mRNA levels by RT-qPCR, as described in Materials and Methods. Data shown are the mean $\pm S D(n=3)$, and the WT level is presented as 1.0. (B) Proto, MgProto, and MgProtoMe levels in wild type and tCHLH plants in the presence of NF. Tetrapyrrole was extracted and quantified by HPLC and normalized to fresh weight, as described in Materials and Methods. Proto (white bar), MgProto (black), and MgProtoMe (gray) levels are presented. Data shown are the mean \pm SD $(n \geqq 3)$. Statistical significance was determined by Student's $t$-test and the symbols are indicated as in Figure 2.

$\mathrm{N}$-terminal or C-terminal half of CHLH is sufficient for ABA response in guard cells or that these regions might interact with the cch (P642L) mutant form of the CHLH protein, partially restoring function. The latter idea is somewhat supported by the report that the $\mathrm{N}$-terminal half of SynCHLH enhances $\mathrm{Mg}$-chelatase activity in vitro, though such an enhancing effect of the C-terminal half has not been reported (Sirijovski et al., 2008). It is intriguing that C-terminal half (tCHLH-I), which lacks the authentic plastid transit peptide, can complement the ABA phenotype of $c c h$. A similar result was reported previously, whereby truncated CHLH consisting of a.a. 311-1381 or 631-1381 (corresponding to tCHLH-I and tCHLH-J) can complement the cch mutation (Wu et al., 2009). These authors showed that GFP-tagged fragments localize to the cytoplasm, and according to their model, the CHLH protein is embedded in the plastid envelope and the $\mathrm{N}$ - and $\mathrm{C}$-terminal regions are exposed on the cytosolic surface of the plastid. Accordingly, C-terminal region interacts with cytosolic components such as WRKY transcription factors, SOAR1 and SnRK2.6/OST1 to induce ABA-responses.

It should be noted that the effect of truncated CHLHs has only been tested in the wild type or cch mutant background (Wu et al., 2009; Shang et al., 2010; this study). Therefore, the alterations caused by tCHLHs might be dependent on the presence of wild type or cch-form $\mathrm{CHLH}$ proteins. We were unable to address this point in view of tCHLHs lack of complementation of the seedling lethal phenotype of a TDNA knockout mutant. Thereafter, structural domains analyses should be carefully performed in combination with other methodologies such as random mutagenesis of the CHLH protein 
and inducible deactivation of CHLH activity (Schlicke et al., 2014).

\section{AUTHOR CONTRIBUTIONS}

NM designed the research. AN, HI, and NM performed research. $\mathrm{HI}$ and NM analyzed data and wrote the article.

\section{ACKNOWLEDGMENTS}

We thank Toshinori Kinoshita and Atsushi Takemiya for providing mutant seeds, Yongwei Zhang for providing SLiCE, Tomomi Suzuki for helpful discussions, and Gabriela ToledoOrtiz for critical reading the manuscript. This work was supported by JSPS KAKENHI Grants (Numbers JP 24570046 and JP 21570039) to NM and a Grant-in-Aid for Scientific Research on Innovative Areas (No. 22120002) and a Grant-in-Aid for Scientific Research (B) (No. 15H04389) from the Japan Society for the Promotion of Science to AN.

\section{SUPPLEMENTARY MATERIAL}

The Supplementary Material for this article can be found online at: http://journal.frontiersin.org/article/10.3389/fpls.2016. 01650/full\#supplementary-material

\section{Supplementary Figure 1 | Amino acid sequence alignment of Synechocystis and Arabidopsis CHLHs. Amino acid sequences of Synechocystis (BAA17040.1) and Arabidopsis (CAA92802.1) CHLH proteins were aligned using CLUSTALW. The domain structures based on crystallography analysis of SynCHLH are indicated below the sequences respectively as (I) blue, (II) green, (III) orange, (IV) brown, (V) magenta, and (VI) purple lines (Chen et al., 2015). The amino acid positions that are substituted in gun5 mutants are indicated by triangles with the allele names.}

\section{Supplementary Figure 2 | Comparison of chlorophyll, tetrapyrrole intermediates and Lhcb1*2 mRNA levels in wild type (WT) and gun5 mutants grown in the absence of NF. (A) Chlorophyll accumulation. Plants were grown on MS medium supplemented with $2 \%$ sucrose for 5 days under continuous white light $\left(100 \mu \mathrm{mol} / \mathrm{m}^{2} / \mathrm{s}\right)$. Chlorophyll a is presented as a dark-gray bar and chlorophyll b as a light-gray bar, respectively. Data shown are the mean \pm $\mathrm{SD}(n=3)$. (B) Proto, MgProto, and MgProtoMe levels. Plants were grown as described in (A). Tetrapyrrole was extracted and quantified by HPLC and normalized to fresh weight, as described in Materials and Methods. Proto (white bar), MgProto (black), and MgProtoMe (gray) levels are presented. Data shown are the mean $\pm \mathrm{SD}(n \geqq 3)$. (C) Lhcb1 mRNA levels. Plants were grown as described in (A). Lhcb1 mRNA levels were quantified and normalized to TUB2 mRNA levels by RT- QPCR, as described in Materials and Methods. Data shown are the mean $\pm \mathrm{SD}(n=3)$, and the WT level is presented as 1.0. Statistical significance was determined by Student's $t$-test and the symbols are indicated as in Figure $\mathbf{2}$ in the main text.}

Supplementary Figure 3 | Correlation between Lhcb1*2 mRNA and tetrapyrrole levels. Tetrapyrrole intermediate and chlorophyll levels are plotted against the Lhcb1 mRNA levels, and Pearson's correlation coefficient $(r)$ and significance probability $(\mathbf{P})$ are presented in the graph. (A-C) Proto, MgProto, and MgProtoMe levels in -NF condition vs. Lhcb1 mRNA levels in +NF condition. (D-F) Proto, MgProto, and MgProtoMe levels in +NF condition vs. Lhcb1 mRNA levels in +NF condition. (G,H) Chlorophyll vs. Lhcb1 mRNA levels in -NF (G) or in $+\mathrm{NF}(\mathbf{H})$, respectively.

Supplementary Figure 4 | Inhibition of light-induced stomatal opening in gun 5 mutants by ABA. Effect of ABA on light-induced stomatal opening in gun 5 mutants showing normal ABA sensitivity. Experiments were performed as described in Figure $\mathbf{3}$ and Materials and Methods. The light and ABA treatment regimes were as follows: red light at $50 \mu \mathrm{mol} / \mathrm{m}^{2} / \mathrm{s}$ in the absence (red bar) or presence (white bar) of $20 \mu \mathrm{M} \mathrm{ABA}$ (+ABA); blue light at $10 \mu \mathrm{mol} / \mathrm{m}^{2} / \mathrm{s}$ with background red light at $50 \mu \mathrm{mol} / \mathrm{m}^{2} / \mathrm{s}$ in the absence (blue) or presence (gray) of $20 \mu$ M ABA. Statistical significance was determined by Student's $t$-test. * $p<$ 0.05 .

Supplementary Figure 5 | Comparison of CHLH protein levels in gun5 mutants. (A) The CHLH protein levels of 4-week-old wild type and gun5 mutant plants grown in soil under long-day condition. CHLH proteins were detected using an anti-CHLH antibody. Total protein $(10 \mu \mathrm{g})$ was applied to each lane. The relative protein levels are indicated above the lanes (WT as 1.0). (B,C) CHLH protein levels in 5-day-old wild type and gun5 mutant seedlings grown on MS medium in the absence (B) or presence (C) of $2.5 \mu \mathrm{M}$ NF. Total protein $(20 \mu \mathrm{g})$ was applied to each lane. The relative protein levels are indicated below the lanes (WT, control as 1.0). The blot image was spliced and re-aligned for visualization.

Supplementary Figure 6 | Inhibition of seed germination and growth in gun5 mutants by ABA. Wild type and gun5 mutant seeds were sown on MS agar medium containing $2 \%(\mathrm{w} / \mathrm{V})$ sucrose in the absence $\mathbf{( A )}$ or presence (B) of $0.5 \mu \mathrm{M}$ ABA, and the plates were incubated for 8 days after stratification as described in Materials and Methods. The scale bar is $5 \mathrm{~mm}$.

Supplementary Figure $7 \mid \mathrm{CHLH}$ mRNA levels in wild type and gun5 mutant alleles. CHLH mRNA levels in wild type and gun5 mutants grown in the absence (A) or presence (B) of NF. CHLH mRNA levels were quantified and normalized to TUB2 mRNA levels by RT-qPCR, as described in Materials and Methods. Data shown are the mean $\pm \mathrm{SD}(n=3)$, and the WT level is presented as 1.0. Statistical significance was determined by Student's $t$-test and the symbols are indicated as in Figure 2 in the main text.

Supplementary Figure 8 | Comparison of CHLH protein levels in tCHLH lines (tCHLH-A, -B, -C, - D) in 5-day-old seedlings. (A,B) CHLH protein levels in 5-day-old wild type, cch and tCHLH-A, -B, -C, -D seedlings grown on MS medium in the absence (A) or presence (B) of $2.5 \mu \mathrm{M}$ NF. Total protein $(20 \mu \mathrm{g})$ was applied to each lane. Proteins were first detected using the anti-CHLH antibody (top), followed by the anti-FLAG antibody (bottom) after stripping the first antibody. RBCL bands on the stained membrane are indicated as loading controls in (A). For (B), RBCL bands are unclear due to the NF treatment, thus anonymous bands are indicated as loading controls. The position of the endogenous $\mathrm{CHLH}$ protein is indicated by an asterisk.

Supplementary Figure 9 | Comparison of CHLH protein levels in tCHLH lines (tCHLH-E, - F, - G, - H, - I) in 5-day-old seedlings. (A,B) CHLH protein levels in 5-day-old wild type, $c c h$ and tCHLH-E, $-F,-G,-H,-I$ seedlings grown on MS medium in the absence (A) or presence (B) of $2.5 \mu \mathrm{M}$ NF. Total protein (20 $\mu \mathrm{g})$ was applied to each lane. Proteins were first detected using the anti-CHLH antibody (top), followed by the anti-FLAG antibody (bottom) after stripping the first antibody. The position of the endogenous $\mathrm{CHLH}$ protein is indicated by an asterisk. The blot image was spliced and re-aligned for visualization. (C,D) $\mathrm{CH} L \mathrm{H}$ protein levels of 5-day-old wild type, cch and $\mathrm{tCH} L \mathrm{H}-\mathrm{H},-\mathrm{I},-\mathrm{J}$ seedlings grown on MS medium in the absence (C) or presence (D) of $2.5 \mu \mathrm{M} \mathrm{NF}$. To enhance detection, approximately $60 \mu \mathrm{g}$ total protein was applied to each lane and detected using the anti-FLAG antibody. Total protein extract $(20 \mu \mathrm{g})$ of tCHLH-D \#4-1 was used as a positive control. In (A,C), RBCL bands on the stained membrane are indicated as loading controls. For (B,C), anonymous bands are indicated as loading controls as described in Supplementary Figure 8.

Supplementary Figure 10 | Comparison of CHLH protein levels in tCHLH lines in 4-week-old plants. (A,B) CHLH protein levels in 4-week-old wild type, cch and $\mathrm{tCH} L \mathrm{H}$ plants grown in soil under long-day conditions. Total protein (20 $\mu \mathrm{g})$ was applied to each lane. Proteins were first detected using the anti-CHLH antibody (top), followed by the anti-FLAG antibody (bottom) after stripping the first antibody. The position of the endogenous CHLH protein is indicated by an asterisk. The blot image was spliced and re-aligned for visualization. RBCL bands on the stained membrane are indicated as loading controls.

Supplementary Figure 11 | Comparison of gun and chlorophyll phenotypes in wild type and tCHLH lines. (A,B,D) Characterization of the $t C H L H-I$ line was performed separately from other $\mathrm{tCH} L \mathrm{H}$ lines due to the limited availability of seeds. (A) Lhcb1 mRNA levels in wild type, cch and tCHLH-I plants in the presence of NF. Lhcb1 mRNA levels were quantified and normalized to TUB2 mRNA levels by RT-qPCR, as described in Materials and 
Methods. Data shown are the mean $\pm \operatorname{SD}(n=3)$, and the WT level is presented as 1.0. (B) Proto, MgProto, and MgProtoMe levels in wild type, cch and $\mathrm{tCHLH}-\mathrm{I}$ plants in the presence of NF. Tetrapyrrole was extracted and quantified by HPLC and normalized to fresh weight, as described in Materials and Methods. Proto (white bar), MgProto (black), and MgProtoMe (gray) levels are presented. Data shown are the mean \pm SD $(n \geqq 3)$. (C,D) Chlorophyll accumulation. Plants were grown on MS medium supplemented with $2 \%$ sucrose for 5 days under continuous white light $\left(100 \mu \mathrm{mol} / \mathrm{m}^{2} / \mathrm{s}\right)$. Chlorophyll a is presented as a dark-gray bar and chlorophyll b as a light-gray bar, respectively. Data shown are the mean values for $(\mathbf{C})(n=2)$, and mean $\pm \mathrm{SD}$ for (D) $(n=3)$. Statistical significance was determined by Student's $t$-test and the symbols are indicated as in Figure $\mathbf{2}$ in the main text.

\section{Supplementary Figure 12 | Comparison of gun and tetrapyrrole} phenotypes in wild type and tCHLH plants grown in the absence of NF. (A,B) Proto, MgProto, and MgProtoMe levels in wild type, cch and tCHLH plants in the absence of NF. (B) Tetrapyrrole levels in $\mathrm{tCH} L \mathrm{H}-\mathrm{I}$ were assessed separately from other lines due to the limited availability of seeds. Data shown are the mean $\pm \mathrm{SD}(n \geqq 3)$. (C,D) Lhcb1 mRNA levels in wild type, cch, CHLH-full and tCHLH plants in the absence of NF. Lhcb1 mRNA levels were quantified and normalized to TUB2 mRNA levels by RT-qPCR. (D) Lhcb1 mRNA levels in $\mathrm{tCHLH}-\mathrm{I}$ were assessed separately from other lines due to the limited availability of seeds. Data shown are the mean $\pm \operatorname{SD}(n=3)$, and the WT level is presented as 1.0. (D) There is no significant difference in the average value between the wild type and tCHLH-I lines. Statistical significance was determined by Student's $t$-test and the symbols are indicated as in Figure 2 in the main text

Supplementary Figure 13 | Inhibition of light-induced stomatal opening in tCHLH lines by ABA. Effect of ABA on light-induced stomatal opening in tCHLH lines. The experiments were performed as described in Figure 3. Light and ABA treatment regimes are as follows: red light at $50 \mu \mathrm{mol} / \mathrm{m}^{2} / \mathrm{s}$ in the absence (red bar); blue light at $10 \mu \mathrm{mol} / \mathrm{m}^{2} / \mathrm{s}$ with background red light at $50 \mu \mathrm{mol} / \mathrm{m}^{2} / \mathrm{s}$ in the absence (blue) or presence (gray) of $20 \mu \mathrm{M}$ ABA. Statistical significance was determined by Student's $t$-test. $* p<0.05$.

Supplementary Figure 14 | Schematic diagrams of the currently proposed functional and structural domain architecture of the CHLH protein, and the positions of the amino acid substitutions and phenotypes in the gun 5 mutants used in this study. (Top) Black and gray boxes represent the $\mathrm{CHLH}$ protein and the transit peptide, respectively. Wild type amino acids and positions and mutant amino acids are indicated. (A) Vertical black and gray lines represent mutants showing strong or moderate phenotypes in the assays indicated in the left, respectively. Asterisks indicate the mutants exhibiting constitutively closed stomatal phenotypes. (B) Green boxes represent the proposed domain structures based on crystallography analysis of SynCHLH (Chen et al., 2015). (C) White boxes represent the proposed regions responsible for ABA binding, the transmembrane domain and the WRKY interaction domain (Wu et al., 2009; Shang et al., 2010).

\section{Supplementary Table 1 | Primers used in this study.}

\section{REFERENCES}

Adams, N. B. P., Marklew, C. J., Qian, P., Brindley, A. A., Davison, P. A., Bullough, P. A., et al. (2014). Structural and functional consequences of removing the $\mathrm{N}$-terminal domain from the magnesium chelatase $\mathrm{ChlH}$ subunit of Thermosynechococcus elongatus. Biochem. J. 464, 315-322. doi: 10.1042/BJ20140463

Adhikari, N. D., Froehlich, J. E., Strand, D. D., Buck, S. M., Kramer, D. M., and Larkin, R. M. (2011). GUN4-porphyrin complexes bind the ChlH/GUN5 subunit of Mg-Chelatase and promote chlorophyll biosynthesis in Arabidopsis. Plant Cell 23, 1449-1467. doi: 10.1105/tpc.110.082503

Alawady, A. E., and Grimm, B. (2005). Tobacco Mg protoporphyrin IX methyltransferase is involved in inverse activation of $\mathrm{Mg}$ porphyrin and protoheme synthesis. Plant J. 41, 282-290. doi: 10.1111/j.1365313X.2004.02291.x

Chan, K. X., Phua, S. Y., Crisp, P., McQuinn, R., and Pogson, B. J. (2016). Learning the languages of the chloroplast: retrograde signaling and beyond. Annu. Rev. Plant Biol. 67, 25-53. doi: 10.1146/annurev-arplant-043015-111854

Chen, H., Nelson, R. S., and Sherwood, J. L. (1994). Enhanced recovery of transformants of Agrobacterium tumefaciens after freeze-thaw transformation and drug selection. Biotechniques 16, 664-668, 670.

Chen, X., Pu, H., Fang, Y., Wang, X., Zhao, S., Lin, Y., et al. (2015). Crystal structure of the catalytic subunit of magnesium chelatase. Nat. Plants 1, 15125. doi: $10.1038 /$ nplants.2015.125

Clough, S. J., and Bent, A. F. (1998). Floral dip: a simplified method for Agrobacterium-mediated transformation of Arabidopsis thaliana. Plant J. 16, 735-743. doi: 10.1046/j.1365-313x.1998.00343.x

Cutler, S. R., Rodriguez, P. L., Finkelstein, R. R., and Abrams, S. R. (2010). Abscisic acid: emergence of a core signaling network. Annu. Rev. Plant Biol. 61, 651-679. doi: 10.1146/annurev-arplant-042809-112122

Davison, P. A., and Hunter, C. N. (2011). Abolition of magnesium chelatase activity by the gun 5 mutation and reversal by Gun4. FEBS Lett. 585, 183-186. doi: 10.1016/j.febslet.2010.11.037

Du, S. Y., Zhang, X. F., Lu, Z., Xin, Q., Wu, Z., Jiang, T., et al. (2012). Roles of the different components of magnesium chelatase in abscisic acid signal transduction. Plant Mol. Biol. 80, 519-537. doi: 10.1007/s11103-0129965-3

Gibson, L. C., Willows, R. D., Kannangara, C. G., von Wettstein, D., and Hunter, C. N. (1995). Magnesium-protoporphyrin chelatase of Rhodobacter sphaeroides: reconstitution of activity by combining the products of the bchH, -I, and -D

genes expressed in Escherichia coli. Proc. Natl. Acad. Sci. U.S.A. 92, 1941-1944 doi: 10.1073/pnas.92.6.1941

Gray, J. C., Sornarajah, R., Zabron, A. A., Duckett, C. M., and Khan, M. S. (1995). "Chloroplast control of nuclear gene expression," in Photosynthesis: From Light to Biosphere, Vol. 3, ed P. Mathis (Dordrecht: Kluwer), 543-550.

Horton, R. M., Hunt, H. D., Ho, S. N., Pullen, J. K., and Pease, L. R. (1989). Engineering hybrid genes without the use of restriction enzymes: gene splicing by overlap extension. Gene 77, 61-68. doi: 10.1016/0378-1119(89)90359-4

Huang, Y. S., and Li, H. M. (2009). Arabidopsis CHLI2 can substitute for CHLI1. Plant Physiol. 150, 636-645. doi: 10.1104/pp.109.135368

Ibata, H., Nagatani, A., and Mochizuki, N. (2013). Perforated-tape Epidermal Detachment (PED): a simple and rapid method for isolating epidermal peels from specific areas of Arabidopsis leaves. Plant Biotechnol. 30, 497-502. doi: 10.5511/plantbiotechnology.13.0903b

Inoue, S., Kinoshita, T., Matsumoto, M., Nakayama, K. I., Doi, M., and Shimazaki, K. (2008). Blue light-induced autophosphorylation of phototropin is a primary step for signaling. Proc. Natl. Acad. Sci. U.S.A. 105, 5626-5631. doi: 10.1073/pnas.0709189105

Koussevitzky, S., Nott, A., Mockler, T. C., Hong, F., Sachetto-Martins, G., Surpin, M., et al. (2007). Signals from chloroplasts converge to regulate nuclear gene expression. Science 316, 715-719. doi: 10.1126/science.1140516

Larkin, R. M., Alonso, J. M., Ecker, J. R., and Chory, J. (2003). GUN4, a regulator of chlorophyll synthesis and intracellular signaling. Science 299, 902-906. doi: $10.1126 /$ science. 1079978

Liang, S., Lu, K., Wu, Z., Jiang, S. C., Yu, Y. T., Bi, C., et al. (2015). A link between magnesium-chelatase $\mathrm{H}$ subunit and sucrose nonfermenting 1 (SNF1)-related protein kinase SnRK2.6/OST1 in Arabidopsis guard cell signalling in response to abscisic acid. J. Exp. Bot. 66, 6355-6369. doi: 10.1093/jxb/erv341

McBride, K. E., and Summerfelt, K. R. (1990). Improved binary vectors for Agrobacterium-mediated plant transformation. Plant Mol. Biol. 14, 269-276. doi: 10.1007/BF00018567

Mei, C., Jiang, S. C., Lu, Y. F., Wu, F. Q., Yu, Y. T., Liang, S., et al. (2014). Arabidopsis pentatricopeptide repeat protein SOAR1 plays a critical role in abscisic acid signalling. J. Exp. Bot. 65, 5317-5330. doi: 10.1093/jxb/eru293

Mochizuki, N., Brusslan, J. A., Larkin, R., Nagatani, A., and Chory, J. (2001). Arabidopsis genomes uncoupled 5 (GUN5) mutant reveals the involvement of Mg-chelatase $\mathrm{H}$ subunit in plastid-to-nucleus signal transduction. Proc. Natl. Acad. Sci. U.S.A. 98, 2053-2058. doi: 10.1073/pnas.98.4.2053

Mochizuki, N., Tanaka, R., Tanaka, A., Masuda, T., and Nagatani, A. (2008). The steady-state level of Mg-protoporphyrin IX is not a determinant of 
plastid-to-nucleus signaling in Arabidopsis. Proc. Natl. Acad. Sci. U.S.A. 105, 15184-15189. doi: 10.1073/pnas.0803245105

Moulin, M., McCormac, A. C., Terry, M. J., and Smith, A. G. (2008). Tetrapyrrole profiling in Arabidopsis seedlings reveals that retrograde plastid nuclear signaling is not due to Mg-protoporphyrin IX accumulation. Proc. Natl. Acad. Sci. U.S.A. 105, 15178-15183. doi: 10.1073/pnas.0803054105

Muller, A. H., and Hansson, M. (2009). The barley magnesium chelatase 150kd subunit is not an abscisic acid receptor. Plant Physiol. 150, 157-166. doi: 10.1104/pp.109.135277

Nakagawa, T., Suzuki, T., Murata, S., Nakamura, S., Hino, T., Maeo, K., et al. (2007). Improved Gateway binary vectors: high-performance vectors for creation of fusion constructs in transgenic analysis of plants. Biosci. Biotechnol. Biochem. 71, 2095-2100. doi: 10.1271/bbb.70216

Porra, R. J., Thompson, W. A., and Kriedemann, P. E. (1989). Determination of accurate extinction coefficients and simultaneous equations for assaying chlorophylls a and b extracted with four different solvents: verification of the concentration of chlorophyll standards by atomic absorption spectroscopy. Biochim. Biophys. Acta Bioenerg. 975, 384-394. doi: 10.1016/S0005-2728(89)80347-0

Qian, P., Marklew, C. J., Viney, J., Davison, P. A., Brindley, A. A., Soderberg, C., et al. (2012). Structure of the cyanobacterial magnesium chelatase $\mathrm{H}$ subunit determined by single particle reconstruction and small-angle X-ray scattering. J. Biol. Chem. 287, 4946-4956. doi: 10.1074/jbc.M111. 308239

Schlicke, H., Hartwig, A. S., Firtzlaff, V., Richter, A. S., Glässer, C., Maier, K., et al. (2014). Induced deactivation of genes encoding chlorophyll biosynthesis enzymes disentangles tetrapyrrole-mediated retrograde signaling. Mol. Plant 7, 1211-1227. doi: 10.1093/mp/ssu034

Shang, Y., Yan, L., Liu, Z. Q., Cao, Z., Mei, C., Xin, Q., et al. (2010). The Mgchelatase $\mathrm{H}$ subunit of Arabidopsis antagonizes a group of WRKY transcription repressors to relieve ABA-responsive genes of inhibition. Plant Cell 22, 1909-1935. doi: 10.1105/tpc.110.073874

Shen, Y. Y., Wang, X. F., Wu, F. Q., Du, S. Y., Cao, Z., Shang, Y., et al. (2006). The Mg-chelatase $\mathrm{H}$ subunit is an abscisic acid receptor. Nature 443, 823-826. doi: 10.1038 /nature 05176

Sirijovski, N., Lundqvist, J., Rosenbäck, M., Elmlund, H., Al-Karadaghi, S., Willows, R. D., et al. (2008). Substrate-binding model of the chlorophyll biosynthetic magnesium chelatase BchH subunit. J. Biol. Chem. 283, 11652-11660. doi: 10.1074/jbc.M709172200

Strand, A., Asami, T., Alonso, J., Ecker, J. R., and Chory, J. (2003). Chloroplast to nucleus communication triggered by accumulation of $\mathrm{Mg}$-protoporphyrinIX. Nature 421, 79-83. doi: 10.1038/nature01204

Susek, R. E., Ausubel, F. M., and Chory, J. (1993). Signal transduction mutants of Arabidopsis uncouple nuclear $\mathrm{CAB}$ and RBCS gene expression from chloroplast development. Cell 74, 787-799. doi: 10.1016/0092-8674(93)9 0459-4

Tadini, L., Pesaresi, P., Kleine, T., Rossi, F., Guljamow, A., Sommer, F., et al. (2016). GUN1 controls accumulation of the plastid ribosomal protein $\mathrm{S} 1$ at the protein level and interacts with proteins involved in plastid protein homeostasis. Plant Physiol. 170, 1817-1830. doi: 10.1104/pp.15.02033

Takemiya, A., Sugiyama, N., Fujimoto, H., Tsutsumi, T., Yamauchi, S., Hiyama, A., et al. (2013). Phosphorylation of BLUS1 kinase by phototropins is a primary step in stomatal opening. Nat. Commun. 4, 2094. doi: 10.1038/ncomms3094

Tanaka, R., Kobayashi, K., and Masuda, T. (2011). Tetrapyrrole metabolism in Arabidopsis thaliana. Arabidopsis Book 9:e0145. doi: 10.1199/tab.0145
Terry, M. J., and Smith, A. G. (2013). A model for tetrapyrrole synthesis as the primary mechanism for plastid-to-nucleus signaling during chloroplast biogenesis. Front. Plant Sci. 4:14. doi: 10.3389/fpls.2013.00014

Tomiyama, M., Inoue, S., Tsuzuki, T., Soda, M., Morimoto, S., Okigaki, Y., et al. (2014). Mg-chelatase I subunit 1 and Mg-protoporphyrin IX methyltransferase affect the stomatal aperture in Arabidopsis thaliana. J. Plant Res. 127, 553-563. doi: 10.1007/s10265-014-0636-0

Tsuzuki, T., Takahashi, K., Inoue, S., Okigaki, Y., Tomiyama, M., Hossain, M. A., et al. (2011). Mg-chelatase $\mathrm{H}$ subunit affects ABA signaling in stomatal guard cells, but is not an ABA receptor in Arabidopsis thaliana. J. Plant Res. 124, 527-538. doi: 10.1007/s10265-011-0426-x

Tsuzuki, T., Takahashi, K., Tomiyama, M., Inoue, S., and Kinoshita, T. (2013). Overexpression of the $\mathrm{Mg}$-chelatase $\mathrm{H}$ subunit in guard cells confers drought tolerance via promotion of stomatal closure in Arabidopsis thaliana. Front. Plant Sci. 4:440. doi: 10.3389/fpls.2013.00440

Willows, R. D., Gibson, L. C., Kanangara, C. G., Hunter, C. N., and von Wettstein, D. (1996). Three separate proteins constitute the magnesium chelatase of Rhodobacter sphaeroides. Eur. J. Biochem. 235, 438-443. doi: 10.1111/j.14321033.1996.00438.x

Woodson, J. D., Perez-Ruiz, J. M., and Chory, J. (2011). Heme synthesis by plastid ferrochelatase I regulates nuclear gene expression in plants. Curr. Biol. 21, 897-903. doi: 10.1016/j.cub.2011.04.004

Wu, F. Q., Xin, Q., Cao, Z., Liu, Z. Q., Du, S. Y., Mei, C., et al. (2009). The $\mathrm{Mg}$-chelatase $\mathrm{H}$ subunit binds abscisic acid and functions in abscisic acid signaling: new evidence in Arabidopsis. Plant Physiol. 150, 1940-1954. doi: 10.1104/pp.109.140731

Yamaguchi, R., Nakamura, M., Mochizuki, N., Kay, S. A., and Nagatani, A. (1999). Light-dependent translocation of a phytochrome B-GFP fusion protein to the nucleus in transgenic Arabidopsis. J. Cell Biol. 145, 437-445. doi: 10.1083/jcb.145.3.437

Zhang, D. P., Wu, Z. Y., Li, X. Y., and Zhao, Z. X. (2002). Purification and identification of a 42-kilodalton abscisic acid-specific-binding protein from epidermis of broad bean leaves. Plant Physiol. 128, 714-725. doi: 10.1104/pp.010531

Zhang, X. F., Jiang, T., Wu, Z., Du, S. Y., Yu, Y. T., Jiang, S. C., et al. (2013). Cochaperonin CPN20 negatively regulates abscisic acid signaling in Arabidopsis. Plant Mol. Biol. 83, 205-218. doi: 10.1007/s11103-013-0082-8

Zhang, Y., Werling, U., and Edelmann, W. (2012). SLiCE: a novel bacterial cell extract-based DNA cloning method. Nucleic Acids Res. 40:e55. doi: 10.1093/nar/gkr1288

Zhang, Z. W., Yuan, S., Feng, H., Xu, F., Cheng, J., Shang, J., et al. (2011). Transient accumulation of $\mathrm{Mg}$-protoporphyrin IX regulates expression of PhANGs - New evidence for the signaling role of tetrapyrroles in mature Arabidopsis plants. $J$. Plant Physiol. 168, 714-721. doi: 10.1016/j.jplph.2010.10.016

Conflict of Interest Statement: The authors declare that the research was conducted in the absence of any commercial or financial relationships that could be construed as a potential conflict of interest.

Copyright (c) 2016 Ibata, Nagatani and Mochizuki. This is an open-access article distributed under the terms of the Creative Commons Attribution License (CC BY). The use, distribution or reproduction in other forums is permitted, provided the original author(s) or licensor are credited and that the original publication in this journal is cited, in accordance with accepted academic practice. No use, distribution or reproduction is permitted which does not comply with these terms. 